\title{
Structure of a glutamate transporter homologue from Pyrococcus horikoshit
}

\author{
Dinesh Yernool ${ }^{1 \star}$, Olga Boudker ${ }^{1,2 *}$, Yan Jin $^{2}$ \& Eric Gouaux ${ }^{1,2}$ \\ ${ }^{1}$ Department of Biochemistry and Molecular Biophysics and ${ }^{2}$ Howard Hughes Medical Institute, Columbia University, 650 West $168 t h$ Street, New York, New York \\ 10032, USA \\ ${ }^{*}$ These authors contributed equally to this work
}

Glutamate transporters are integral membrane proteins that catalyse the concentrative uptake of glutamate from the synapse to intracellular spaces by harnessing pre-existing ion gradients. In the central nervous system glutamate transporters are essential for normal development and function, and are implicated in stroke, epilepsy and neurodegenerative diseases. Here we present the crystal structure of a eukaryotic glutamate transporter homologue from Pyrococcus horikoshii. The transporter is a bowl-shaped trimer with a solvent-filled extracellular basin extending halfway across the membrane bilayer. At the bottom of the basin are three independent binding sites, each cradled by two helical hairpins, reaching from opposite sides of the membrane. We propose that transport of glutamate is achieved by movements of the hairpins that allow alternating access to either side of the membrane.

The chemical synapse is a central site for communication between neurons in the human brain. At chemical synapses an action potential promotes the release of neurotransmitter, increasing the concentration of transmitter $10^{3}-10^{4}$-fold in the synaptic cleft. The neurotransmitter opens ligand-gated ion channels, resulting in depolarization of the postsynaptic neuron and generation of a postsynaptic receptor potential. At many synapses, integral membrane transport proteins clear the transmitter from the synaptic cleft, reducing the concentration of transmitter to basal level, thereby readying the synapse for a subsequent cycle of activation ${ }^{1}$.

Glutamatergic synapses are the chemical synapses that mediate the majority of fast excitatory neurotransmission ${ }^{2}$. Essential for normal development and function, the glutamatergic synapse is a linchpin for learning and memory, and dysfunction at these synapses is implicated in a wide range of nervous system diseases and injuries, including schizophrenia, depression and stroke ${ }^{3}$. Rapid clearance of glutamate from the synapse by high-affinity, sodium-dependent transporters is required for normal excitatory neurotransmission and prevention of glutamate-induced excitotoxicity ${ }^{4,5}$

The high-affinity, sodium-dependent glutamate transporters are members of a family of integral membrane transport proteins that include five eukaryotic glutamate transporters, two eukaryotic neutral amino acid transporters, and a large number of bacterial amino acid and dicarboxylic acid transporters ${ }^{5,6}$. Eukaryotic members of this transporter family have an essential role in the nervous system and they function in many other organs, including the heart, kidney and intestine $e^{7}$. In prokaryotes, these transporters carry out the concentrative uptake of metabolites across the membrane by the co-transport of protons and/or sodium ions ${ }^{6}$. Physiological studies have elaborated the ion stoichiometry of eukaryotic glutamate transporters, showing that glutamate uptake is coupled to the co-transport of three sodium ions and one proton, and to the counter-transport of one potassium ion ${ }^{8}$. Notably, eukaryotic glutamate transporters also possess a thermodynamically uncoupled, glutamate-gated chloride conductance, illuminating their dual roles as secondary transporters and ligand-gated ion channels ${ }^{9}$.

Prokaryotic and eukaryotic glutamate and neutral amino acid transporters possess significant amino acid sequence relationships throughout their entire polypeptides ${ }^{6}$ (Fig. 1). Residues in the carboxy-terminal half of eukaryotic and prokaryotic transporters are crucial for substrate binding, substrate transport and ion coupling (for recent reviews, see refs 10-12), whereas residues in the amino-terminal portion of the eukaryotic transporters are implicated in the thermodynamically uncoupled chloride flux ${ }^{13}$. Determination of the transmembrane topology of glutamate transporters has been fraught with uncertainty, and there are multiple models, each possessing non-canonical elements of transmembrane protein structure ${ }^{14-20}$. Thus, despite the wealth of functional data on glutamate transporters, there is no understanding of their threedimensional architecture or molecular transport mechanism.

\section{Structure determination}

To reveal the molecular architecture of glutamate transporters, and to provide an atomic basis for a mechanism of substrate and ion transport, we crystallized a glutamate transporter homologue from P. horikoshii ( $\mathrm{Glt}_{\mathrm{Ph}}$; Supplementary Table S1), which shares $37 \%$ amino acid identity with human excitatory amino acid transporter 2 (hEAAT2). In the course of our crystallization trials we found that a multiple point mutant of Glt $\mathrm{Ph}_{\mathrm{Ph}}$, in which seven His residues were introduced into non-conserved sites on predicted loops $\left(\mathrm{Glt}_{\mathrm{Ph}} 7 \mathrm{H}\right)$, was expressed at higher levels and crystallized more readily than the wild-type protein (Fig. 1).

Crystals of $\mathrm{Glt}_{\mathrm{Ph}} 7 \mathrm{H}$ diffract to $3.2 \AA$ along $c^{\star}$ and $3.8 \AA$ along $a^{*}$ and belong to the space group $P 6_{1}$ (Table 1). Initial phases were obtained from a $6 \AA$ resolution multiwavelength anomalous diffraction (MAD) experiment ${ }^{21}$ using a platinum derivative. Six heavy atom sites, arranged as two sites per protomer, confirmed the trimeric subunit stoichiometry of prokaryotic transporters ${ }^{22}$ and defined a three-fold axis of non-crystallographic symmetry (NCS). The MAD phases were applied to a native data set and extended to $3.5 \AA$ resolution using $\mathrm{DM}^{23}$. To assist in model building we exploited the presence of 16 Met residues per protomer by determining selenium sites from anomalous difference Fourier maps of a selenomethionine derivative. In addition, we substituted Met residues into six sites with ultimately each transmembrane segment containing one or two Met residues (Fig. 1; see also Supplementary Table S2). Iterative cycles of model building and refinement were then carried out. The final model contains all amino acid residues except for $11 \mathrm{~N}$-terminal and $6 \mathrm{C}$-terminal residues, and a number of disordered side chains modelled as Ala.

We also collected data from an isomorphous crystal of the wildtype Glt $t_{\mathrm{Ph}}$ protein that diffracted to $4.1 \AA$ (Supplementary Table S2). The phases from $\mathrm{Glt}_{\mathrm{Ph}} 7 \mathrm{H}$ were applied to the $\mathrm{Glt}_{\mathrm{Ph}}$ data, followed by density modification and crystallographic refinement. The partially 
refined structure and accompanying electron density maps did not reveal any significant differences between Glt $_{\mathrm{Ph}} \mathrm{H} 7$ and $\mathrm{Glt}_{\mathrm{Ph}}$.

\section{Trimer architecture}

The Glt $t_{\mathrm{Ph}} \mathrm{H} 7$ trimer is bowl-shaped with a concave aqueous basin facing the extracellular solution and a pointed base facing the cytoplasm (Fig. 2). The three-fold NCS axis is perpendicular to the membrane, and when viewed in this orientation the trimer has a triangular shape with sides of $\sim 80 \AA$. Viewed parallel to the membrane, the trimer is $\sim 65 \AA$ in height, with the transmembrane-spanning portion of the transporter lying approximately in the middle, thus indicating that the transporter protrudes about $15 \AA$ from each side of the membrane bilayer. The basin is as large as $50 \AA$ in diameter and $30 \AA$ in depth, and dips far into the membrane plane. Because the extracellular basin is deep and its surface hydrophilic, it allows aqueous, bulk solution to reach the midpoint of the membrane bilayer (Fig. 2d).
There are prominent crevices between the subunits on the lipidexposed surface of the trimer (Fig. 2). Transmembrane 4 (TM4) is located in this crevice and participates in intersubunit contacts. TM1 and TM6 form an additional crevice on the lipid-exposed face of each subunit. In electron density maps we see non-protein density features in both crevices that may be bound lipid or detergent molecules. These crevices allow lipid molecules to access helical hairpins 1 and 2 (HP1, HP2), which are key functional regions of the transporter, and may provide a structural basis for understanding how lipids modulate the activity of bacterial and eukaryotic transporters ${ }^{24-26}$.

\section{Protomer structure}

$\mathrm{Glt}_{\mathrm{Ph}} 7 \mathrm{H}$ protomers are shaped like pointed wedges where the wide ends define the extracellular rim of the basin and the pointed tips come together at the three-fold axis, forming the bottom of the basin and, on the intracellular face, a cone-shaped structure.

a

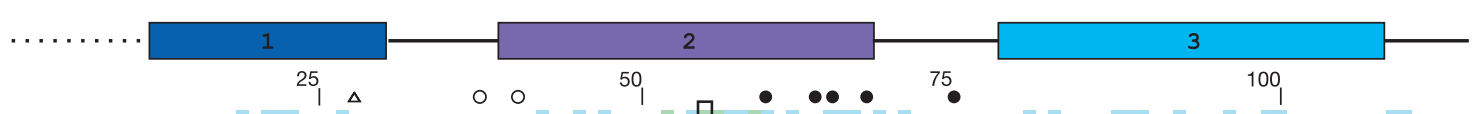

Gl $t_{p h} 1$ MGLYRKYIEYPVLQKILIGLILGAIVGLILGHYG-YADAVKTYVKPFGDLFVRLIRMLVMPIVFASLVVGAASISPAR-LGRVGVKIVVYYLLTSAFAVTLGIIMARLFNPGAGIHL Gl $t_{B s} \quad 1 \quad$ MRK I GLAWQIFIGLILGIIVGAIFYGNP--- - KVATYLQPIGDIFLRLIKMIVIPIVISSLVVGVASVGDLKKLGKLGGKTIIYFEIITTIAIVVGLLAANIFQPGTGVNM EAAT1 39 EDVKSYLFRNAFVLLTVTAVIVGTILGFTLRPYR - MSYREVKYFSFPGELLMRML QMLVLPLIISSLVTGMAAL-DSKASGKMGMRAVVYYMTTTIIAVVIGIIIVIIIHPGKGTKE

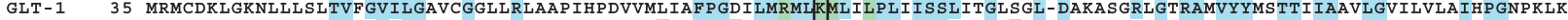
EAAT3 9 CEWKRFLKNNWVLLSTVAAVVLGITTGVLVREHSNLSTLEKFYFAFPGEILMRMLKLIILPLIISSMITGVAAL-DSNVSGKIGLRAVVYYFCTTLIAVILGIVLVVS IKPGVTQKV ASCT1 33 RRCAGFLRRQALVLLTVSGVLAGAGLGAALRGLS-LSRTQVTYLAFPGEMLLRMLRMIILPLVVCSLVSGAASL-DASCLGRLGGIAVAYFGLTTLSASALAVALAFIIKPGSGAQT

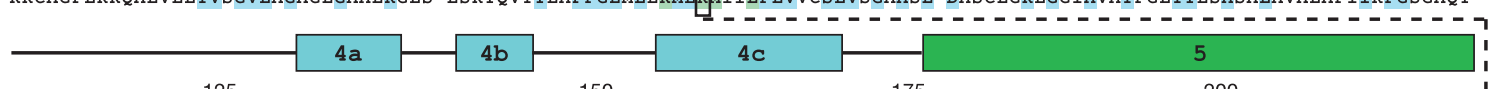

$1250 \Delta \circ \Delta$

150

175 I $\nabla \nabla$

200

Gl $t_{\mathrm{ph}} 116$ AVGG-- - - - - QQFQPKQAPPLVKILLDIVPTNPFGALANG--_- - QVLPTIFFAIILGIAITYLMNSENEKVRKSAETLLDAINGLAEAMYKIVNGVMQYAPIGVFALIAYVMA Glt $t_{B s} 108$ KSLE-KTDIQSYVDTTNEVQHHSMVETFVNIVPKNIFESLTKG- - - - DMLPIIFFSVMFGLGVAAIG- - - - - EKGKPVLQFFQGTAEAMFYVTNQIMKFAPFGVFALIGVTVS I EAAT1 154 NMH-- - - - REGKIVRVTAADAFLDLIRNMFP PNLVEACFKQ-XXX-NALGLVVFSMCFGFVIGNMK - - - - EQGQALREFFDSLNEAIMRLVAVIMWYAPVGILFLIAGKIV I GLT - 1151 KQLG--- - PGKKNDEVSSLDAFLDLIRNLFPENLVQACFQQ-XXX-NVLGLIGFFIAFGIAMGKMG--- - - EQAKLMVEFFNILNEIVMKLVIMIMWYSPLGIACLICGKII I ASCT1 148 LQSSDLGLEDSGPPPVPKETVDSFLDLARNLFPSNLVVAAFRT-XXX-NILGLVLFALVLGVALKKLG-_... - - SEGEDLIRFFNSLNEATMVLVSWIMWYVPVGIMFLVGSKIV
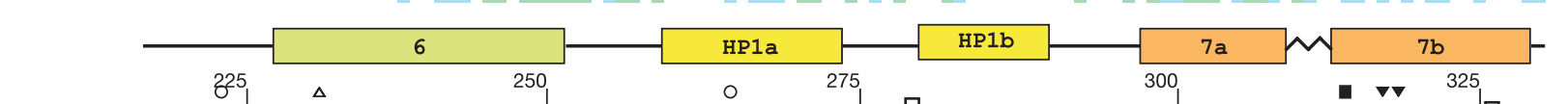

$\mathrm{Gl}_{\mathrm{Ph}} 219$ EQG--VKVVGELAKVTAAVYVGLTLQILLVYFVLLKIY-GIDPISFIKKAKDAMLTAFVTRSSSSTLPVTMRVAKE-MGISEGIYSFTLPLGATINMDGTALYQGVCTFFIANALG Glt 211 KFG--VESLIPLSKLVIVVYATMVFFIFVVLGGVAKLF-GINIFHIIKILKDELILAYSTASSETVLPKIMEKME-NFGCPKAITSFVIPTGYSFNLDGSTLYQALAAIFIA RLYG EAAT1 303 EMEDMGVIGGQLAMYTVTVIVGLLIHAVIVLPLIYFLVTRKNPWVFIGGLLQALITALGTSSS SATLPITFKCLEENNGVDKRVTRFVLPVGATINMD GTALYEALAAIF TA VVNN

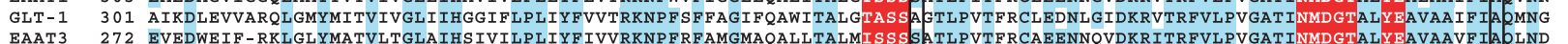
ASCT1 283 EMKDIIVLVTSLGKYIFASILGHVIHGGIVLPLIYFVFTRKNPFRFLLGLLAPFATAFATCSSSATLP SMMKCIEENNGVDKRISRFILPIGATVNMDGAAIFOCVAAVFIARLNN

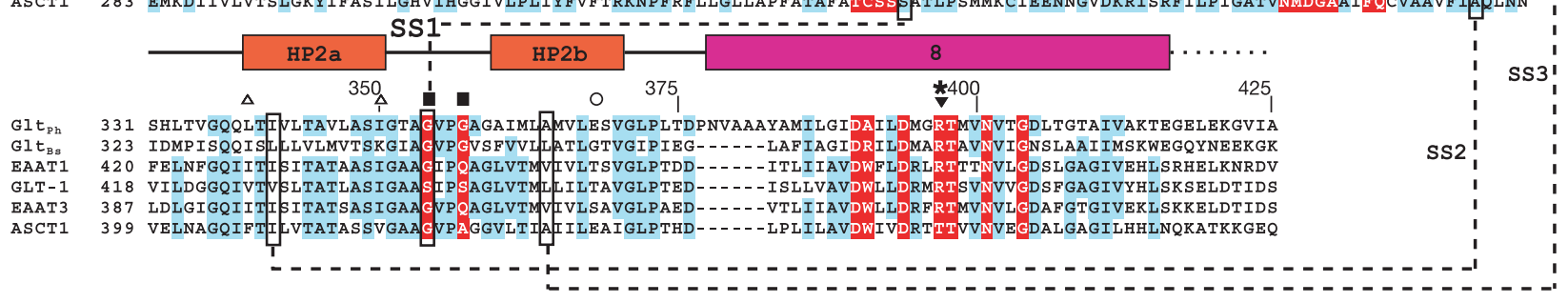
b

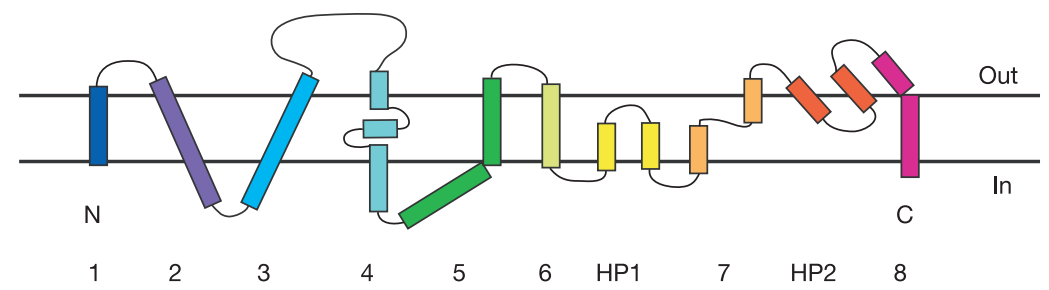

Figure 1 Sequence alignment of Gltph, glutamate and neutral amino acid transporters. a, Boxes above the alignment correspond to $\alpha$-helices and are colour-coded according to Fig. 3. Dotted lines represent residues disordered in the crystal structure. Sequence colouring highlights regions of high homology (blue), intersubunit contacts seen in the crystal structure (green) and residues implicated in glutamate transport (red). Filled symbols above the sequences mark residues involved in glutamate $\gamma$-carboxylate binding (star), sodium binding (squares), potassium coupling (inverted triangles) and chloride conductance (circles). Open symbols mark the histidine point mutants (circles), the methionine mutants (triangles) and the double cysteine mutant (inverted triangles).
Residues in eukaryotic transporters that form disulphide bonds when mutated to cysteines are boxed and the bonds are indicated by dashed lines ${ }^{13,29}$. Insertions in eukaryotic transporters between helices $4 \mathrm{~b}$ and $4 \mathrm{c}$ are not included and are marked by XXX; the longer $\mathrm{N}$ and $\mathrm{C}$ termini of eukaryotic transporters are also not included. Amino acid sequences are: P. horikoshii Gltph (NP_143181), B. stearothermophilus Glt ${ }_{\mathrm{Bs}}$ (P24943); human EAAT1 (P43003); rat GLT-1 (P31596); human EAAT3 (AAH37310); human ASCT1 (NP_003029). The alignment was made using ClustalW ${ }^{50}$ and adjusted manually. b. Schematic representation of Gltph transmembrane topology. 


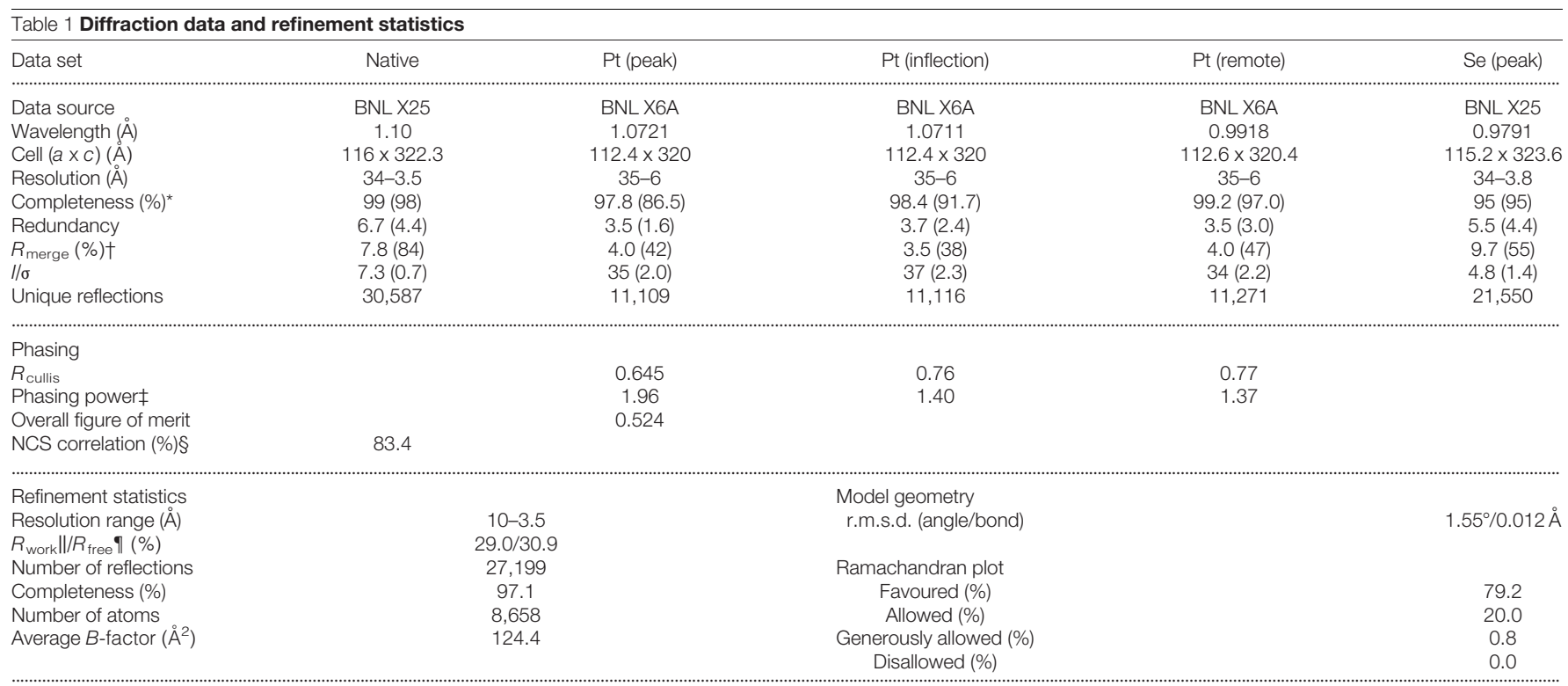

*Values for the highest resolution shell are shown in parentheses and the shells are $3.7-3.5 \AA, 6.2-6.0 \AA$ and $4.0-3.8 \AA$ for the native, Pt and Se data sets, respectively.

$\dagger R_{\text {merge }}=\Sigma\left|/_{\mathrm{hkl}}-\left\langle I_{\mathrm{hkl}}\right\rangle\right| / \Sigma\left(I_{\mathrm{hkl}}\right)$, where $I_{\mathrm{hkl}}$ is the integrated intensity of the given reflection.

$\ddagger$ Phasing power, $\Sigma\left|F_{H}\right| / \Sigma|E|$, was calculated using SHARP.

§Correlation between the electron density areas related by non-crystallographic symmetry.

$\| R_{\text {work }}=\left(\Sigma\left|F_{\mathrm{o}}-F_{\mathrm{c}}\right|\right) / \Sigma F_{\mathrm{o}}$, where $F_{\mathrm{o}}$ and $F_{\mathrm{c}}$ are observed and calculated structure factors.

१ Five per cent of data were excluded from the refinement to calculate $R_{\text {free }}$
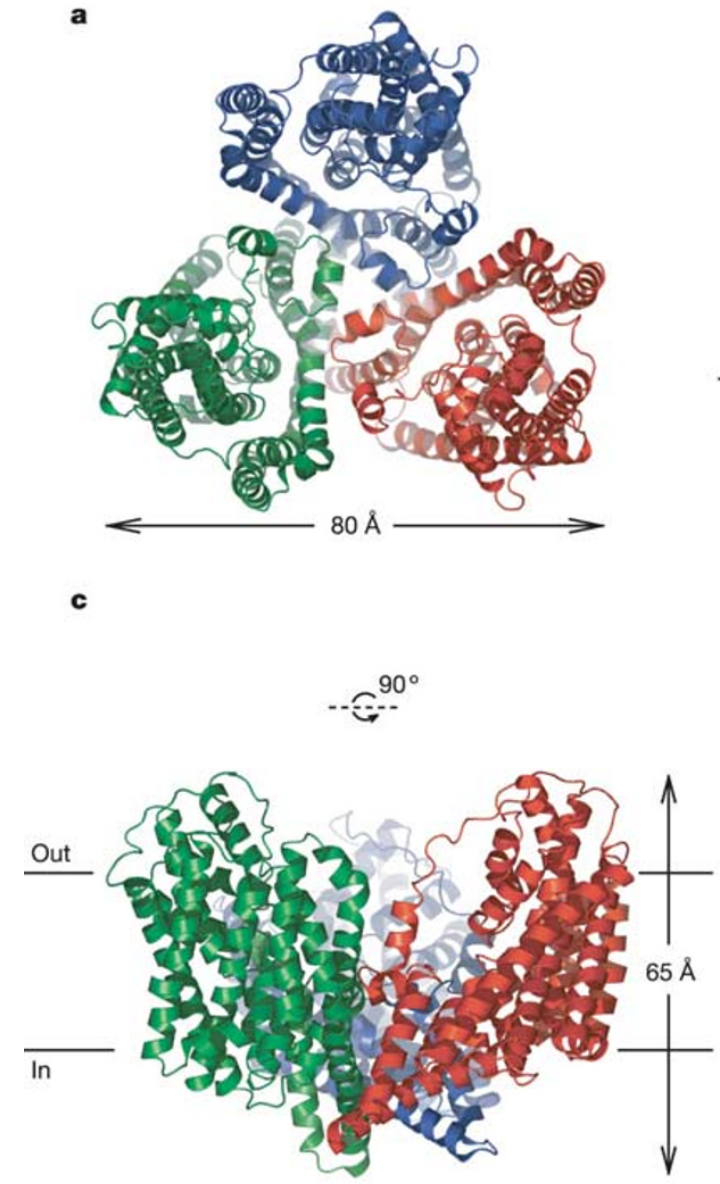

Figure 2 Structure of $\mathrm{Glt}_{\mathrm{ph}} \mathrm{H} 7$. a, Ribbon representation of the trimer, in which the protomers are red, blue and green, viewed from the extracellular side of the membrane. b. View of the trimer from the cytoplasm, showing the locations of crevice 1 , between subunits, and crevice 2, between transmembranes 1 and 6 of each subunit. c. View of the

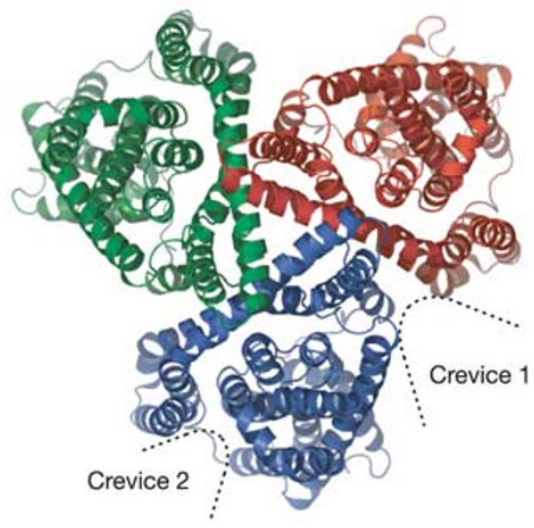

d

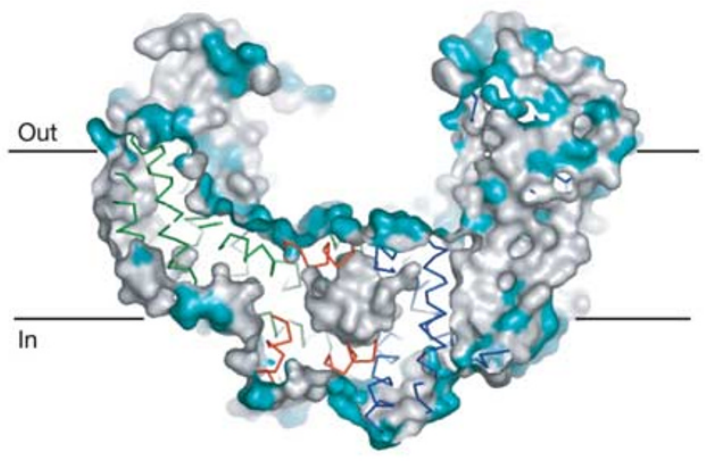

trimer parallel to the membrane. d, Surface representation of the trimer sliced through the centre of the basin. Polar and apolar residues are coloured cyan and white, respectively. The boundaries of the lipid bilayer are indicated in $\mathbf{c}$ and $\mathbf{d}$, using the hydrophobic residues on TM1 as a reference. 
Each protomer has eight primarily $\alpha$-helical transmembrane segments (TMs 1-8) and two helical hairpins (HPs 1-2; Figs 1 and 3). Transmembrane segments 1-6 form a distorted cylinder-shaped motif-the N-terminal cylinder-whose outer surface mediates all of the intersubunit contacts in the trimer. The $\mathrm{C}$-terminal half of the protein-TM7, TM8, HP1 and HP2, implicated in substrate transport-is secured within the $\mathrm{N}$-terminal cylinder, suggesting that each subunit has an independent substrate transport pathway.

The fold of a Glt $\mathrm{Ph}_{\mathrm{p}} 7 \mathrm{H}$ protomer is, to the best of our knowledge, novel and is composed of a number of unusual elements of secondary structure. In particular, TM2, TM3 and TM5 are up to 49 residues in length and are tilted from the membrane normal by as much as $45^{\circ}$. The long, protease-sensitive and proline-rich 'linker' that connects TM3 and TM4 (refs 22, 27) arches from one side of the $\mathrm{N}$-terminal cylinder to the other, over the top of HP2 and TM7 and TM8, spans a distance of about $60 \AA$, and makes only a few contacts with other portions of the subunit. TM4 is composed of multiple elements, has a corkscrew-like, helix-turn-helix-turn-helix structure and forms key subunit-subunit contacts on the threefold axis.

The C-terminal portion of the protomer includes essential elements of the transport machinery. Helical hairpin 1 (HP1) is a helix-turn-helix structure that begins on the cytoplasmic surface of the trimer and is buried within the $\mathrm{N}$-terminal cylinder, reaching up
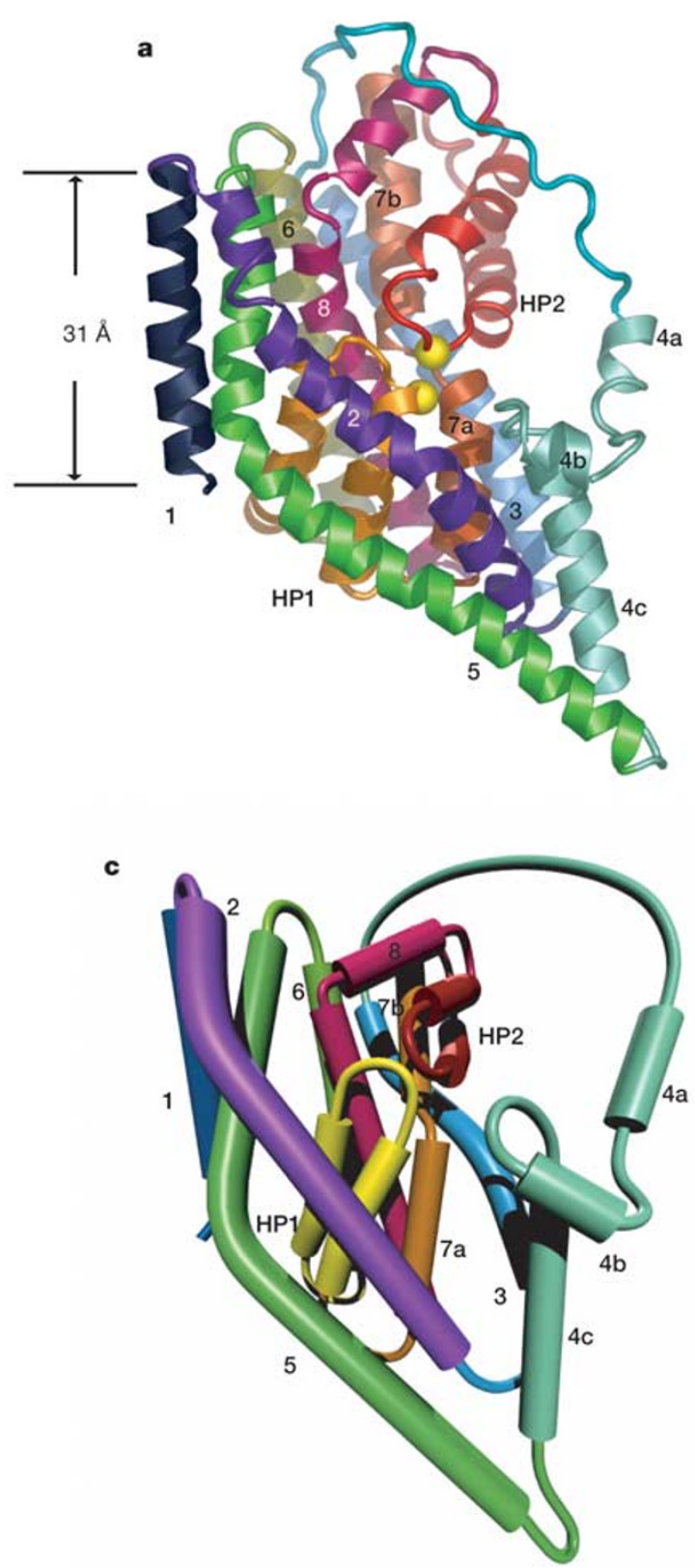

Figure 3 Fold of a Glt $\mathrm{ph}_{\mathrm{H}} \mathrm{H} 7$ protomer. a, Ribbon representation of the protomer viewed in the plane of the membrane in which the transmembrane helices (1-8) and hairpins (HP1, HP2) are labelled and in different colours. The $\alpha$-carbon atoms of Ser 279 (HP1) and Gly 354 (HP2) are defined by yellow spheres, which are equivalent to Ala 364 and Ser 440

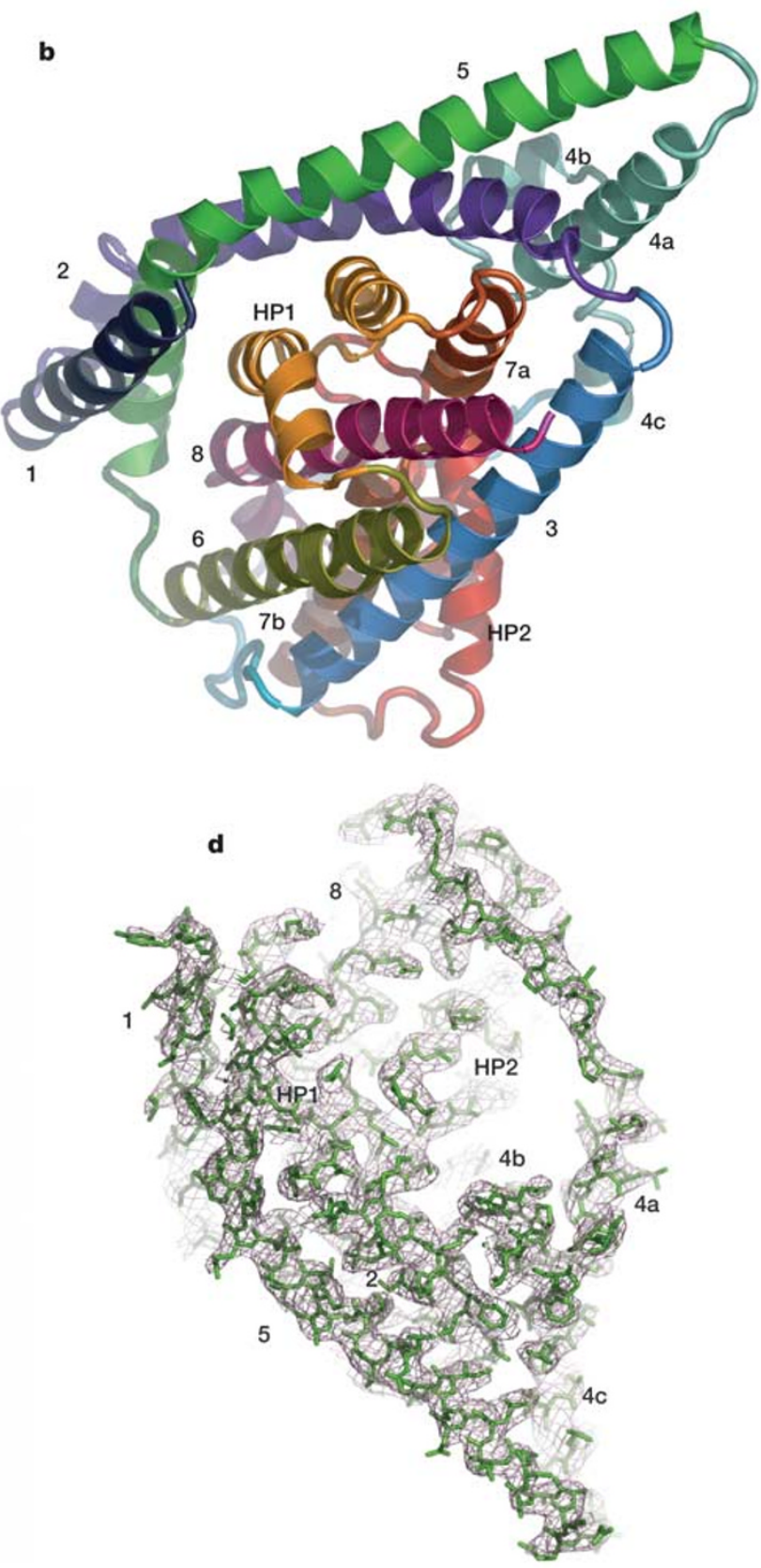

of GLT-1 (ref. 29). b, View of the protomer from the cytoplasm. c, Schematic representation of the protomer fold. $\mathbf{d}$, Slice of electron density from a $2 F_{0}-F_{c}$ map, contoured at $1 \sigma$, overlaying a stick model of a protomer. 
to the bottom of the extracellular basin. A conserved serine-rich motif located in the loop of HP1 tiles part of the basin bottom and is partially exposed to the extracellular solution, in agreement with previous chemical modification experiments ${ }^{14,16,18}$. Passing through the middle of the N-terminal cylinder is TM7, an unusual transmembrane structure with two helical segments, $7 \mathrm{a}$ and $7 \mathrm{~b}$, whose helical axes are parallel but displaced by a conserved, three-residue motif that forms a $\beta$-bridge.

Helical hairpin 2 (HP2) is another key element of the transport machinery and like HP1 it is composed of a helix-turn-helix motif. However, the context of HP2 is different; it is situated almost parallel to the membrane plane, with a large fraction of its surface solvent-exposed and facing the extracellular basin. At the tip of HP2 there is a conserved proline (Pro 356 in $\mathrm{Glt}_{\mathrm{Ph}} 7 \mathrm{H}$ ) in van der Waals contact with the serine-rich motif at the tip of HP1. Connected to HP2 is TM8, an amphipathic $\alpha$-helical segment that runs through the middle of the $\mathrm{N}$-terminal cylinder and has been suggested to line a portion of the substrate transport pathway ${ }^{28}$.

HP1 and HP2, together with flanking regions from TM7 and TM8, are structurally related and can be superimposed with a root mean square deviation (r.m.s.d.) of $2.4 \AA$ (Supplementary Fig. S1), even though HP1 and HP2 have no significant amino acid sequence identity. Most importantly, the tips of HP1 and HP2 meet at the bottom of the basin, about halfway across the membrane bilayer. The apposition of HP1 and HP2 was foreshadowed by experiments on the rat glutamate transporter GLT-1, in which Ala 364 and Ser 440 were changed to cysteine. This double cysteine mutant of GLT-1 was active in glutamate transport only under reducing conditions, suggesting that a disulphide bond formed between residues 364 and 440 under oxidizing conditions ${ }^{29}$. In Glt $_{\mathrm{Ph}} 7 \mathrm{H}$ the residues equivalent to Ala 364 and Ser 440 of GLT-1 are Ser 279 and Gly 354, respectively, they map to the tips of HP1 and HP2 and are sufficiently close to form a disulphide bond (Fig. 3a; see also Supplementary Fig. S1).

\section{Subunit interface and oligomerization state}

The Glt $_{\mathrm{Ph}} 7 \mathrm{H}$ protomers share substantial intersubunit interfaces with each subunit burying $\sim 2,045 \AA^{2}$ in a trimerization motif composed of TM2, TM4 and TM5. On the cytoplasmic face of Glt $_{\mathrm{Ph}} 7 \mathrm{H}$ the $\mathrm{C}$-terminal portion of TM4c and the N-terminal end of TM5 form a bundle crossing or a 'smoke-hole' (Figs 2b and 4a). On the extracellular side of the transporter the TM4b helices define a whorl around the symmetry axis (Fig. 2a) while TM2 cements intersubunit contacts between TM4c/TM5 in one subunit and the corkscrew/TM4c of its neighbour. Eukaryotic transporters have insertions of 32-55 amino acids between TM4b and TM4c (Fig. 1), which may be accommodated within the basin.

Viewed along the membrane normal TM4b, TM4c, TM5 and TM2 form a distinct trimerization domain (Figs 2a, b and 4). At the centre of the domain, around the three-fold axis, is a vestibule of $\sim 400 \AA^{3}$ (Fig. 2d). The residues lining the vestibule are hydrophobic and even though there are positive electron density features in the cavity, identification of the chemical composition of the bound molecule(s) is not possible at this moderate resolution. There are small portals into the vestibule, from both the basin and the cytoplasmic smoke-hole, but the diameters of the openings are only $2-3 \AA$. Given its nonpolar character and limited access, the vestibule is unlikely to serve as a permeation pathway for ions or substrates.

To confirm our assignment of key subunit-subunit contacts, we designed a double cysteine mutant (Ser 179 changed to Cys/Asp 185 changed to Cys, referred to hereafter as S179C/D185C) to form an intersubunit disulphide bond linking subunits together (Fig. 4a). The double cysteine mutant, when treated with copper phenanthroline, forms a $138-\mathrm{kDa}$ trimer, as determined by mass spectrometry (Fig. 4b). Because these two non-native cysteine residues readily form a disulphide-linked trimer, our crystal structure is relevant to the oligomerization state of the transporter in a non-crystalline environment.

To determine the subunit stoichiometry of eukaryotic transporters we expressed the human EAAT2 transporter in HEK 293 cells as a fusion with green fluorescent protein (hEAAT2-GFP). After purification by size exclusion chromatography and glutaraldehyde cross-linking, hEAAT2-GFP forms a pattern of cross-linked species that is consistent with a trimer (Fig. 4c). Therefore, on the basis of the $\mathrm{Glt}_{\mathrm{Ph}} 7 \mathrm{H}$ structure, the conservation of residues in subunit interfaces, the cross-linking of hEAAT2-GFP and previous work from this group ${ }^{22}$ and others ${ }^{30}$, proteins in the prokaryotic and eukaryotic glutamate transporter family are trimers.

\section{Substrate-binding site}

A telltale clue to the binding site for substrate along the transport pathway comes from conspicuous non-protein electron density near the interface between HP1 and HP2 (Fig. 5). This electron density feature, approximately the size of a glutamate molecule, cannot be modelled as a protein side chain, and after real space three-fold averaging is greater than $6 \sigma$. Because we included L-glutamate at all stages of purification and crystallization, it is possible that the electron density is a glutamate molecule. However, owing to the modest resolution of our diffraction data we cannot unambiguously identify the molecule(s). We also have been unable to elicit transport activity from $\mathrm{Glt}_{\mathrm{Ph}}$, using a number of methods, suggesting that $\mathrm{Glt}_{\mathrm{Ph}}$ may require archaeal lipids or an elevated

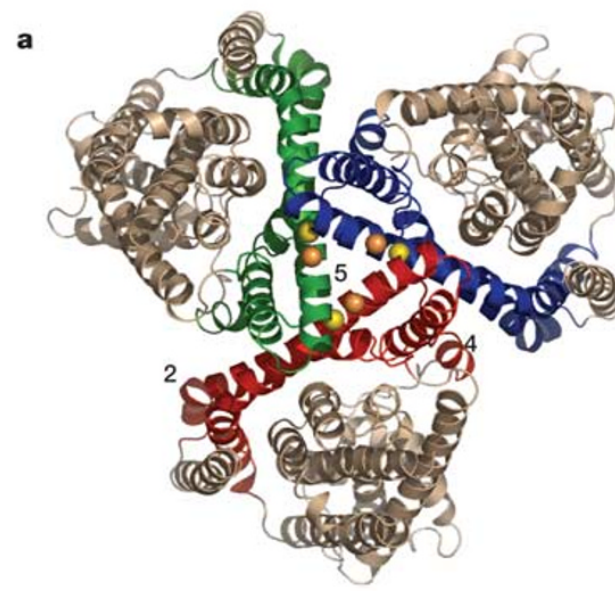

b

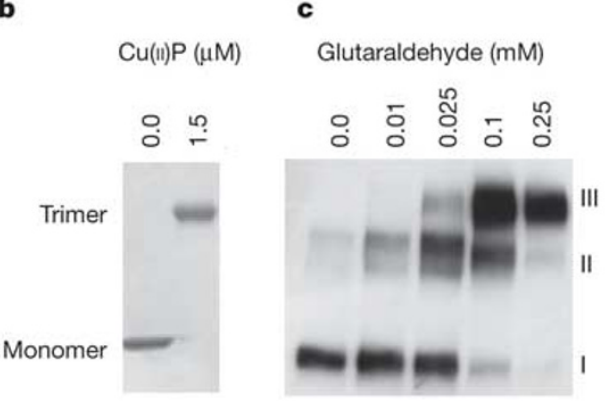

Figure 4 Oligomerization state of prokaryotic and eukaryotic glutamate transporters. a, Transmembrane segments 2, 4 and 5 form a trimerization domain and these three segments are red, blue and green in each of the three subunits, viewed from the cytoplasm. The yellow and orange spheres indicate the sulphur atoms in a model of the Ser 179 and Asp 185 double cysteine mutant. b. SDS-PAGE analysis of the Glt ${ }_{p h} \mathrm{H} 7$ S179C/D185C mutant, untreated and treated with copper phenanthroline. c, Western blot of hEAAT2-GFP cross-linked with glutaraldehyde. Bands I, II and III correspond to monomer, dimer and trimer. 
temperature for functional activity. Nevertheless, the presence of this prominent electron density feature, combined with its provocative location, is suggestive of a substrate-binding site in $\mathrm{Glt}_{\mathrm{Ph}} \mathrm{H} 7$.

The location of the substrate-binding site is noteworthy because the amino acids that surround the site are conserved across transporter homologues and are critical to functional activity

a

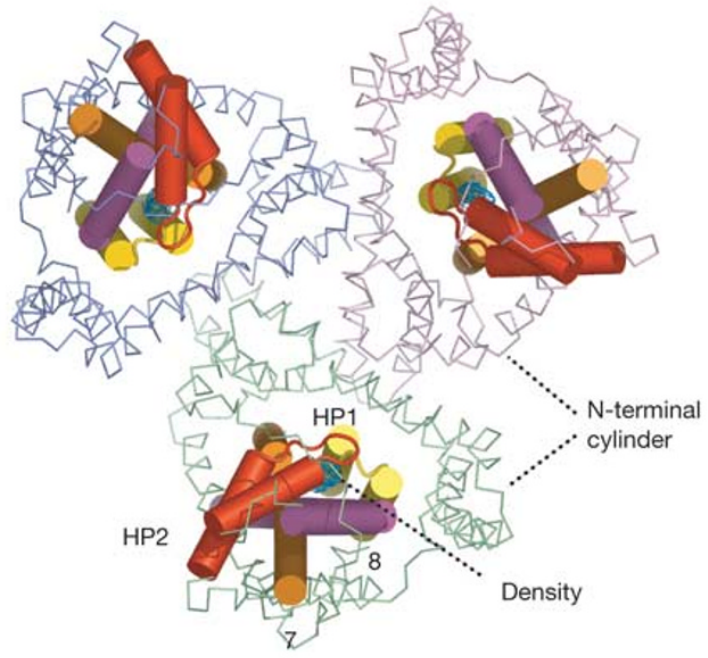

b

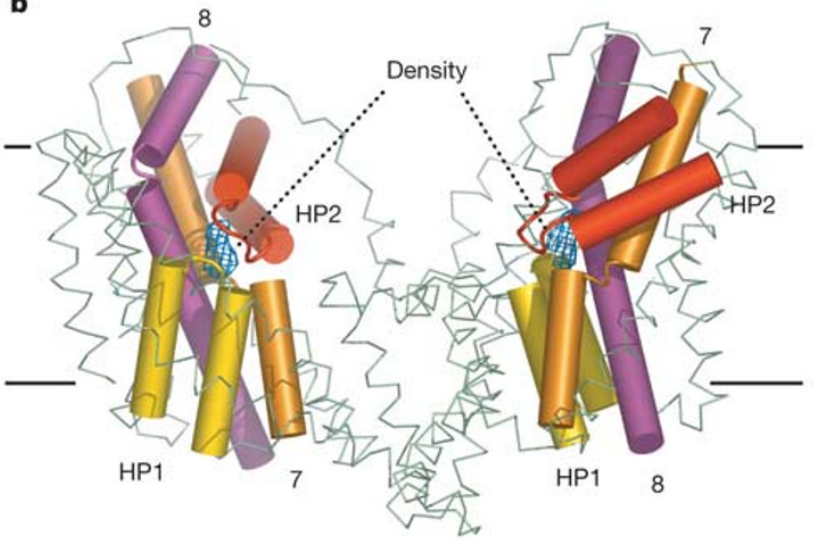

c

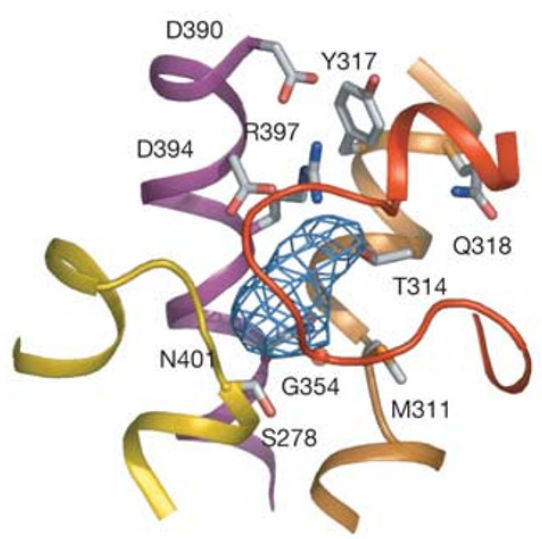

Figure $\mathbf{5}$ Substrate-binding site is located between the tips of HP1 and HP2. $\mathbf{a}, \mathbf{b}$, Shown are $\mathbf{a}, \mathrm{Glt} \mathrm{ph}_{\mathrm{H}} \mathrm{H}$ trimer viewed from the extracellular space and $\mathbf{b}$, two subunits viewed parallel to the membrane plane with $\mathrm{N}$-terminal cylinders represented by an $\alpha$-carbon trace and with HP1, TM7, HP2 and TM8 drawn as cylinders and coloured according to Fig. 3. At the tips of HP1 and HP2 is the non-protein electron density (blue mesh) that defines the substrate-binding site, from a three-fold averaged, $F_{0}-F_{\mathrm{c}}$ map contoured at $4 \sigma . \mathbf{c}$, A close-up view of the substrate-binding site, with residues implicated in glutamate and ion binding shown in stick representation, together with the non-protein electron density, contoured and coloured as in $\mathbf{a}$ and $\mathbf{b}$.
(Figs 1 and 5). The binding site, of which there is one per subunit, is located below the basin, and is covered by the tip of HP2. In eukaryotic transporters HP2 contains residues that are important for sodium binding. In particular, Ser 440 and Ser 443 in GLT-1, which are equivalent to Gly 354 and Gly 357 in $\mathrm{Glt}_{\mathrm{Ph}} 7 \mathrm{H}$, are important for sodium selectivity of the transporter ${ }^{31}$. Gly 354 and Gly 357 in Glt $\mathrm{Ph}_{\mathrm{Ph}} 7 \mathrm{H}$ flank the tip of HP2 and are within $5 \AA$ of the substrate-binding site.

Bounding the other sides of the binding site are the conserved serine residues at the tip of HP1, the $\beta$-bridge of TM7, and a polar portion of TM8 (Fig. 5). In TM7 the 'NMDGT' motif contributes to the substrate-binding pocket: the side chains of Met 311 and Thr 314 point towards the binding pocket while Asn 310 and Asp 312 point away from the binding pocket, interacting with each other and with residues in TM3, TM6 and TM8. We suggest that the interactions of Asn 310 and Asp 312 stabilize the $\beta$-bridge structure and the binding pocket. Emphasizing the importance of the NMDGT motif, previous studies have shown that conservative point mutants in this region are non-functional ${ }^{14}$. In the $\mathrm{Glt}_{\mathrm{Ph}} 7 \mathrm{H}$ structure, the conserved residues Asp 394, Arg 397, Thr 398 and Asn 401 are on the polar face of the amphipathic TM8 and are positioned to form numerous interactions with the substratebinding site (Fig. 5; see also Supplementary Fig. S2).

In eukaryotic glutamate transporters the arginine equivalent to 397 in $\mathrm{Glt}_{\mathrm{Ph}} 7 \mathrm{H}$ confers specificity to substrates with $\beta$ - and $\gamma$ carboxy groups, and mutation of the arginine to a neutral residue results in a transporter that preferentially transports neutral amino acids and that no longer counter-transports potassium ${ }^{32}$ (Fig. 1; see also Supplementary S2). Two residues implicated in potassium binding and counter-transport in eukaryotic transporters are in contact with or close to Arg 397. The first is Tyr 317 (ref. 33), a conserved residue in TM7, which is involved in a $\pi$-cation interaction with Arg 397. The second residue is Gln 318, near Arg 397, which in eukaryotic transporters is a glutamate residue crucial to potassium coupling ${ }^{34}$. In the $\mathrm{Glt}_{\mathrm{Ph}} 7 \mathrm{H}$ structure we see that Arg 397 is poised to interact with the $\gamma$-carboxy group of glutamate. Even though we do not know precisely how Tyr 317 and Gln 318 couple ion binding to substrate transport, the $\mathrm{Glt}_{\mathrm{Ph}} \mathrm{H} 7$ structure demonstrates that residues involved in substrate and ion binding are close in space.

\section{Mechanism}

The alternating access mechanism ${ }^{35}$ is a simple model by which to understand the activity of glutamate transporters. In this model an intramembranous substrate-binding site is flanked by two gates that allow alternating access of the substrate to either the extracellular or intracellular solution. Here, we suggest the locations and structural features of the gates, substrate-binding site and transport pathway in the $\mathrm{Glt}_{\mathrm{Ph}} \mathrm{H} 7$ protein (Fig. 6).

Perhaps the most striking feature of the $\mathrm{Glt}_{\mathrm{Ph}} \mathrm{H} 7$ structure is the aqueous basin that allows for substrates and ions to access binding sites approximately halfway across the membrane, directly from bulk solution. Substrate-binding sites are located $\sim 5 \AA$ beneath the basin bottom and are secured underneath the tips of HP2. We suggest that HP2 comprises the extracellular gate. Directly under the binding pocket are HP1, TM7a and the C-terminal part of TM8, and we speculate that HP1 forms the intracellular gate because movement of HP1 relative to TM7 and TM8 would open an aqueous pathway from the substrate-binding site to the cytoplasm. Accordingly, in the Bacillus stearothermophilus glutamate transporter, residues that map to one face of TM8 and the serine-rich region of HP1 are accessible from intracellular solution ${ }^{18,28}$. Moreover, in the Glt $_{\mathrm{Ph}} \mathrm{H} 7$ structure there are small cavities along the HP1 and TM8 interface, suggesting that changes in the packing of the helices are plausible.

We propose that the $\mathrm{Glt}_{\mathrm{Ph}} 7 \mathrm{H}$ structure represents a bound state of the transporter with both gates closed. However, without 


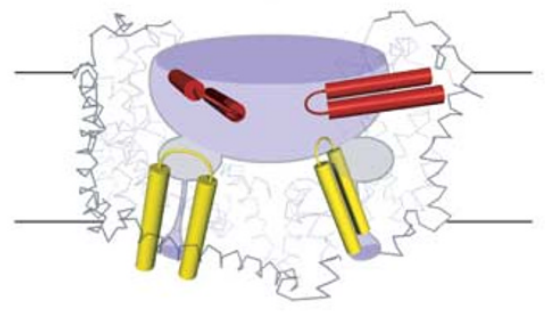

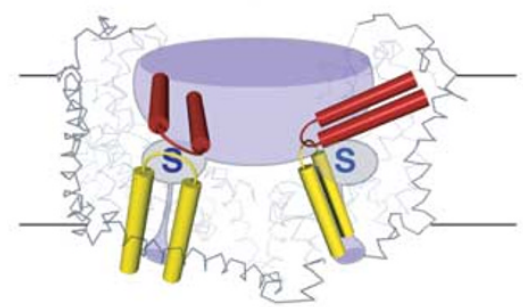

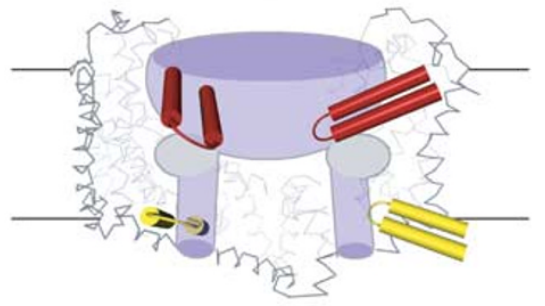

Figure 6 Trimer architecture and mechanism of transport. Glutamate transporters have a large aqueous basin at the bottom of which are located three substrate-binding sites. Here, two of the three substrate-binding sites and transport pathways are shown. Access to the substrate-binding site (shown in grey), from extracellular or intracellular solution, is mediated by HP2 (red) or HP1 (yellow), respectively. a, HP2 is in an 'open' conformation, providing access to the binding site from the extracellular basin. $\mathbf{b}$. Bound state of the transporter observed in the Gltph $\mathrm{H} 7$ structure, where access to the binding site is blocked by HP1 and HP2. The substrate and co-transported ions are represented by the letter S. c, Movement of HP1 out of the protomer core, towards the cytoplasm and away from the three-fold axis, opens a transport pathway from the substrate-binding site to the cytoplasm. structures of specific functional states, we can only speculate on the conformational transitions that occur during transport. Nevertheless, biochemical experiments suggest that HP2 undergoes substrate-dependent conformational changes. For example, the solvent accessibilities of residues in HP2 and TM7 are modulated by glutamate and sodium in eukaryotic transporters ${ }^{36,37}$. Furthermore, fluorescence experiments on hEAAT3 demonstrate that the loop connecting HP2 to TM8 undergoes changes in environment upon glutamate and sodium binding ${ }^{38}$. We therefore suggest that opening of the extracellular gate involves movement of the HP2 'flipper', perhaps allowing HP2 to pack against and stabilize the TM3-TM4 loop. Consistently, protease sensitivity of the TM3-TM4 loop in GLT-1 is increased in the presence of sodium and glutamate ${ }^{27}$.

Even though HP1 and HP2 harbour a marked structural similarity they are located in different protein contexts and therefore the conformational changes they undergo during gating, as well as the chemical cues that activate gating, are probably distinct. To open the intracellular gate we suggest that HP1 moves vertically towards the cytoplasm and laterally into crevice 2 (Figs $2 \mathrm{~b}$ and $6 \mathrm{c}$ ), thereby creating a substrate transport pathway along the polar face of TM8 and rendering the serine-rich region of HP1 accessible to the cytoplasm.

When the intracellular gate is open we suggest that HP2 moves towards the centre of the trimer, occupying the space vacated by the tip of HP1, thereby preventing the formation of an open transmembrane pore. Indeed, movement of HP2 is consistent with the observation that in human EAAT1 a cysteine introduced into HP2 forms a disulphide bond with a cysteine in TM2 (ref. 13); the equivalent residues in $\mathrm{Glt}_{\mathrm{Ph}} \mathrm{H} 7$ are separated by $\sim 20 \AA$. Indeed, chemical modification of cysteines on the surface of HP2 arrests transport but not substrate binding ${ }^{39-41}$, suggesting that HP2 may participate in packing interactions different from those observed in the crystal structure. Finally, the intracellular accessibility of the Ala 432 to Cys mutant in GLT-1, which provided the basis for a proposed second re-entrant loop in glutamate transporters ${ }^{15}$, is inconsistent with the position of $\mathrm{HP} 2$ in the $\mathrm{Glt}_{\mathrm{Ph}} \mathrm{H} 7$ structure because the equivalent residue, Ala 345, is located in the middle of HP2a and not at the tip of HP2. We suggest that movement of HP2 towards the substrate-binding site could expose Ala 345 to the intracellular solution and 'seal' the transport pathway.

\section{Discussion}

The architecture of glutamate transporters is well suited for the rapid binding of glutamate in synapses. The large aqueous basin allows transmitter to diffuse, through bulk solution, to readily accessible binding sites halfway across the membrane bilayer. Once bound, rearrangements of the cytoplasmic HP1, and perhaps additional elements of structure, open a pathway through each subunit to the cytoplasm. Although the $\mathrm{Glt}_{\mathrm{Ph}} \mathrm{H} 7$ structure defines the gates that allow alternating access of the binding site to either side of the membrane, many important questions remain unanswered, including the location of ion binding sites, the molecular mechanism coupling ion and substrate binding, the location of the chloride permeation pathway and, most importantly, the conformational changes that accompany each step in the transport cycle.

\section{Methods}

\section{Protein preparation}

Unlabelled Glt $t_{\mathrm{ph}}$ and Glt $\mathrm{ph}_{\mathrm{h}} \mathrm{H} 7$ were expressed as $\mathrm{His}_{8}$ fusion proteins, using the pBAD24 vector and Escherichia coli Top 10 cells $^{42}$, and both proteins were expressed and purified as described previously ${ }^{22}$. Purified protein was dialysed against a crystallization buffer containing (in mM): 10 HEPES, $25 \mathrm{NaCl}, 25 \mathrm{KCl}, 1 \mathrm{EDTA}, 5 \mathrm{~L}$-glutamate and $7 \beta$-decyl maltoside. Selenomethionine-substituted proteins were expressed in LMG194 cells, and purified in the presence of $2 \mathrm{mM} \beta$-mercaptoethanol. Selenium incorporation, as determined by mass spectrometry, was $>95 \%$.

\section{Crystallization}

Hexagonal rod crystals were grown by vapour diffusion at $4{ }^{\circ} \mathrm{C}$ by mixing equal volumes of protein $\left(7-10 \mathrm{mg} \mathrm{ml}^{-1}\right)$ and reservoir solution containing 14-18\% PEG 1000, $100 \mathrm{mM}$ $\mathrm{Li}_{2} \mathrm{SO}_{4}, 50 \mathrm{mM}$ citric acid, $50 \mathrm{mM} \mathrm{Na} \mathrm{HPO}_{4}$. Prior to flash-cooling in liquid nitrogen, crystals were cryo-protected using a reservoir solution adjusted to 30\% PEG 1000 with 5\% glycerol. The platinum derivative was prepared by soaking crystals in a solution containing $50 \mathrm{mM} \mathrm{K}_{2} \mathrm{Pt}\left(\mathrm{NO}_{2}\right)_{4}$ for $6 \mathrm{~h}$ followed by a $1 \mathrm{~h}$ back-soak.

\section{Structure determination}

Diffraction data sets were indexed, integrated and scaled using HKL2000 (ref. 43) and CCP4 programs. For the Pt MAD data set, initial heavy atom sites were found using Solve ${ }^{44}$ and were refined with $\mathrm{SHARP}^{45}$. MAD phases to $8 \mathrm{~A}$ were applied to the native data set and gradually extended to $3.5 \AA$ using the three-fold averaging, solvent flattening and histogram matching in $\mathrm{DM}^{46}$. An initial model was built using the program $\mathrm{O}^{47}$ and refinement was carried out using REFMAC ${ }^{23}$ and $\mathrm{CNS}^{48}$ with tight three-fold NCS restraints. To determine selenium atom positions in selenomethionine derivatives, anomalous difference maps were calculated using density-modified phases. Because the V231M mutant was non-isomorphous, the initial phases were obtained by molecular replacement using $\mathrm{AMoRe}^{49}$.

\section{Cross-linking}

The Glt $\mathrm{Ph}_{\mathrm{Ph}} 7 \mathrm{H}$ double mutant, $\mathrm{S} 179 \mathrm{C} / \mathrm{D} 185 \mathrm{C}$, was expressed and purified as described above, and either left untreated or treated with $1.5 \mu \mathrm{M}$ of $\mathrm{Cu}$ (II) (1,10-phenantroline) 3 . The samples were analysed by SDS-polyacrylamide gel electrophoresis (PAGE), and by mass spectrometry, under non-reducing conditions.

From a stable HEK 293 cell line expressing hEAAT2-GFP-His $s_{8}$ we determined that the fusion construct was active in ${ }^{3} \mathrm{H}$-glutamate uptake using standard procedures ${ }^{14}$. For cross-linking experiments, membranes were solubilized using $\beta$-dodecyl maltoside and the transporter was partially purified by size exclusion chromatography. The GFP fluorescent peak was subsequently cross-linked with glutaraldehyde at $25^{\circ} \mathrm{C}$ for $30 \mathrm{~min}$ and the reactions were quenched using $150 \mathrm{mM}$ Tris- $\mathrm{HCl}, \mathrm{pH}$ 7.5. The extent of crosslinking was evaluated by western blotting using an anti-His antibody.

Received 12 August; accepted 15 September 2004; doi:10.1038/nature03018.

1. Clements, J. D. Transmitter timecourse in the synaptic cleft: its role in central synaptic function Trends Neurosci. 19, 163-171 (1996).

2. Wheal, H. \& Thomson, A. (eds) Excitatory Amino Acids and Synaptic Transmission (Academic, San Diego, California, 1995). 
3. Dingledine, R., Borges, K., Bowie, D. \& Traynelis, S. F. The glutamate receptor ion channels. Pharmacol. Rev. 51, 7-61 (1999).

4. Bergles, D. E., Diamond, J. S. \& Jahr, C. E. Clearance of glutamate inside the synapse and beyond. Curr. Opin. Neurobiol. 9, 293-298 (1999).

5. Danbolt, N. C. Glutamate uptake. Prog. Neurobiol. 65, 1-105 (2001)

6. Slotboom, D. J., Konings, W. N. \& Lolkema, J. S. Structural features of the glutamate transporter family. Microbiol. Mol. Biol. Rev. 63, 293-307 (1999).

7. Kanai, Y. \& Hediger, M. A. The glutamate and neutral amino acid transporter family: physiological and pharmacological implications. Eur. J. Pharmacol. 479, 237-247 (2003).

8. Zerangue, N. \& Kavanaugh, M. P. Flux coupling in a neuronal glutamate transporter. Nature 383, 634-637 (1996).

9. Fairman, W. A., Vandenberg, R. J., Arriza, J. L., Kavanaugh, M. P. \& Amara, S. G. An excitatory aminoacid transporter with properties of a ligand-gated chloride channel. Nature 375, 599-603 (1995).

10. Amara, S. G. \& Fontana, A. C. Excitatory amino acid transporters: keeping up with glutamate. Neurochem. Int. 41, 313-318 (2002).

11. Kanner, B. I. \& Borre, L. The dual-function glutamate transporters: structure and molecular characterization of the substrate-binding sites. Biochim. Biophys. Acta 1555, 92-95 (2002).

12. Slotboom, D. J., Konings, W. N. \& Lolkema, J. S. Glutamate transporters combine transporter- and channel-like features. Trends Biochem. Sci. 26, 534-539 (2001).

13. Ryan, R. M., Mitrovic, A. D. \& Vandenberg, R. J. The chloride permeation pathway of a glutamate transporter and its proximity to the glutamate translocation pathway. J. Biol. Chem. 279, 20742-2075 (2004).

14. Seal, R. P., Leighton, B. H. \& Amara, S. G. A model for the topology of excitatory amino acid transporters determined by the extracellular accessibility of substituted cysteines. Neuron 25, 695-706 (2000).

15. Grunewald, M., Bendahan, A. \& Kanner, B. I. Biotinylation of single cysteine mutants of the glutamate transporter GLT-1 from rat brain reveals its unusual topology. Neuron 21, 623-632 (1998).

16. Grunewald, M. \& Kanner, B. I. The accessibility of a novel reentrant loop of the glutamate transporte GLT-1 is restricted by its substrate. J. Biol. Chem. 275, 9684-9689 (2000).

17. Slotboom, D. J., Lolkema, J. S. \& Konings, W. N. Membrane topology of the C-terminal half of the neuronal, glial, and bacterial glutamate transporter family. J. Biol. Chem. 271, 31317-31321 (1996).

18. Slotboom, D. J., Sobczak, I., Konings, W. N. \& Lolkema, J. S. A conserved serine-rich stretch in the glutamate transporter family forms a substrate-sensitive reentrant loop. Proc. Natl Acad. Sci. USA 96, 14282-14287 (1999)

19. Wahle, S. \& Stoffel, W. Membrane topology of the high-affinity L-glutamate transporter (GLAST-1) of the central nervous system. J. Cell Biol. 135, 1867-1877 (1996).

20. Jording, D. \& Puhler, A. The membrane topology of the Rhizobium meliloti C4-dicarboxylate permease (DctA) as derived from protein fusions with Escherichia coli K12 alkaline phosphatase (PhoA) and $\beta$-galactosidase (LacZ). Mol. Gen. Genet. 241, 106-114 (1993).

21. Hendrickson, W. A. Determination of macromolecular structures from anomalous diffraction of synchrotron radiation. Science 254, 51-58 (1991).

22. Yernool, D., Boudker, O., Folta-Stogniew, E. \& Gouaux, E. Trimeric subunit stoichiometry of the glutamate transporters from Bacillus caldotenax and Bacillus stearothermophilus. Biochemistry $\mathbf{4 2}$ 12981-12988 (2003)

23. CCP4 Project, Number 4, The CCP4 suite: programs for protein crystallography. Acta Crystallogr. D 50, 760-763 (1994).

24. Tzingounis, A. V., Lin, C.-L., Rothstein, J. D. \& Kavanaugh, M. P. Arachidonic acid activates a proton current in the rat glutamate transporter EAAT4. J. Biol. Chem. 273, 17315-17317 (1998).

25. Fairman, W. A., Sonders, M. S., Murdoch, G. F. \& Amara, S. G. Arachidonic acid elicits a substrategated proton current associated with the glutamate transporter EAAT4. Nature Neurosci. 1, 105-113 (1998).

26. Tolner, B., Ubbink-Kok, T., Poolman, B. \& Konings, W. N. Cation-selectivity of the L-glutamate transporters of Escherichia coli, Bacillus stearothermophilus and Bacillus caldotenax: dependence on the environment in which the proteins are expressed. Mol. Microbiol. 18, 123-133 (1995).

27. Grunewald, M. \& Kanner, B. I. Conformational changes monitored on the glutamate transporter GLT-1 indicate the existence of two neurotransmitter-bound states. J. Biol. Chem. 270, 17017-17024 (1995).

28. Slotboom, D. J., Konings, W. N. \& Lolkema, J. S. Cysteine-scanning mutagenesis reveals a highly amphipathic, pore-lining membrane-spanning helix in the glutamate transporter GltT. J. Biol. Chem 276, 10775-10781 (2001).

29. Brocke, L., Bendahan, A., Grunewald, M. \& Kanner, B. I. Proximity of two oppositely oriented reentrant loops in the glutamate transporter GLT-1 identified by paired cysteine mutagenesis. J. Biol. Chem. 277, 3985-3992 (2002).

30. Gendreau, S. et al. A trimeric quaternary structure is conserved in bacterial and human glutamate transporters. J. Biol. Chem. 279, 39505-39512 (2004).

31. Zhang, Y. \& Kanner, B. I. Two serine residues of the glutamate transporter GLT-1 are crucial for coupling the fluxes of sodium and the neurotransmitter. Proc. Natl Acad. Sci. USA 96, 1710-1715 (1999)

32. Bendahan, A., Armon, A., Madani, N., Kavanaugh, M. P. \& Kanner, B. I. Arginine 447 plays a pivotal role in substrate interactions in a neuronal glutamate transporter. J. Biol. Chem. 275, 37436-37442 (2000)

33. Zhang, Y., Bendahan, A., Zarbiv, R., Kavanaugh, M. P. \& Kanner, B. I. Molecular determinant of ion selectivity of a $\left(\mathrm{Na}^{+}+\mathrm{K}^{+}\right)$-coupled rat brain glutamate transporter. Proc. Natl Acad. Sci. USA 95, 751-755 (1998).

34. Kavanaugh, M. P., Bendahan, A., Zerangue, N., Zhang, Y. \& Kanner, B. I. Mutation of an amino acid residue influencing potassium coupling in the glutamate transporter GLT-1 induces obligate exchange. J. Biol. Chem. 272, 1703-1708 (1997).

35. Jardetzky, O. Simple allosteric model for membrane pumps. Nature 211, 969-970 (1966).

36. Grunewald, M., Menaker, D. \& Kanner, B. I. Cysteine-scanning mutagenesis reveals a conformationally sensitive reentrant pore-loop in the glutamate transporter GLT-1. J. Biol. Chem. 277, 26074-26080 (2002).

37. Leighton, B. H., Seal, R. P., Shimamoto, K. \& Amara, S. G. A hydrophobic domain in glutamate transporters forms an extracellular helix associated with the permeation pathway for substrates. J. Biol. Chem. 277, 29847-29855 (2002).

38. Larsson, H. P., Tzingounis, A. V., Koch, H. P. \& Kavanaugh, M. P. Fluorometric measurements of conformational changes in glutamate transporters. Proc. Natl Acad. Sci. USA 101, 3951-3956 (2004).

39. Borre, L., Kavanaugh, M. P. \& Kanner, B. I. Dynamic equilibrium between coupled and uncoupled modes of a neuronal glutamate transporter. J. Biol. Chem. 277, 13501-13507 (2002)

40. Seal, R. P., Shigeri, Y., Eliasof, S., Leighton, B. H. \& Amara, S. G. Sulfhydryl modification of V449C in the glutamate transporter EAAT1 abolishes substrate transport but not the substrate-gated anion conductance. Proc. Natl Acad. Sci. USA 98, 15324-15329 (2001).

41. Ryan, R. M. \& Vandenberg, R. J. Distinct conformational states mediate the transport and anion channel properties of the glutamate transporter EAAT-1. J. Biol. Chem. 277, 13494-13500 (2002).

42. Guzman, L. M., Belin, D., Carson, M. J. \& Beckwith, J. Tight regulation, modulation, and high-level expression by vectors containing the arabinose PBAD promoter. J. Bacteriol. 177, 4121-4130 (1995).

43. Otwinowski, Z. \& Minor, W. Processing of X-ray diffraction data collected in oscillation mode. Methods Enzymol. 276, 307-326 (1997).

44. Terwilliger, T. C. \& Berendzen, J. Automated MAD and MIR structure solution. Acta Crystallogr. D 55 , 849-861 (1999).

45. de La Fortelle, E. \& Bricogne, G. Maximum-likelihood heavy-atom parameter refinement for multiple isomorphous replacement and multiwavelength anomalous diffraction methods. Methods Enzymol. 276, 472-494 (1997).

46. Cowtan, K. D. Phase combination and cross validation in iterated density-modification calculations. Acta Crystallogr. D 52, 43-48 (1996).

47. Jones, T. A. \& Kjeldgaard, M. Electron-density map interpretation. Methods Enzymol. 277, 173-208 (1997)

48. Brunger, A. T. et al. Crystallography and NMR system: A new software suite for macromolecular structure determination. Acta Crystallogr. D 54, 905-921 (1998).

49. Navaza, J. AMoRe: An automated package for molecular replacement. Acta Crystallogr. A 50, 157-163 (1994).

50. Thompson, J. D., Higgins, D. G. \& Gibson, T. J. CLUSTAL W: Improving the sensitivity of progressive multiple sequence alignment through sequence weighting, position-specific gap penalties and weight matrix choice. Nucleic Acids Res. 22, 4673-4680 (1994).

Supplementary Information accompanies the paper on www.nature.com/nature.

Acknowledgements We appreciate the beamtime, and the assistance of the personnel, a beamlines X4A, X6A, X25 and X26 at the National Synchrotron light source, where all of the diffraction data sets were measured. S. Amara is acknowledged for supplying the hEAAT2 DNA, R. Tsien for the GFP DNA, T. Kawate for the chromatography/fluorimetry set-up, M. Gawinowicz for mass spectrometry, E. Robel for Figs 3c and 6, and R. Ryan, S. Harrison and R. MacKinnon for comments. This work was supported by the Howard Hughes Medical Institute (O.B., E.G., Y.J.) and the NIH (D.Y., O.B., E.G.). D.Y. was also supported by a NIH postdoctoral fellowship. E.G. is an assistant investigator with the Howard Hughes Medical Institute.

Competing interests statement The authors declare that they have no competing financial interests.

Correspondence and requests for materials should be addressed to E.G. (jeg52@columbia.edu). The coordinates for the structure are deposited in the Protein Data Bank under accession code $1 \mathrm{XFH}$. 